\title{
La jurilinguistique: un appui indispensable à la corédaction
}

\author{
Lionel A. Levert
}

Published online: 3 June 2014

(C) The Author(s) 2014. This article is published with open access at Springerlink.com

\begin{abstract}
The advent and expanding role of jurilinguistics as part of the federal legislative process are closely associated with the gradual recognition of the equal authority of the two linguistic versions of federal legislation, as well as the implementation of codrafting as the most effective method of taking into account the equal authority of the two official languages of the country. Jurilinguistics gradually made its way into the federal legislative process starting in the mid 70s and quickly resulted in the establishment of a formal group dealing with French jurilinguistics, comprised of Francophone drafters and jurilinguists. Jurilinguists played a key role in the development of drafting standards for the drafting of a truly authentic French version of federal legislation. In the late 90s, Anglophone jurilinguists were also hired to ensure the highest quality possible for the English version of the federal laws and, along with their Francophone counterparts, to ensure the functional equivalence of the two language versions. The implementation of the departmental policy on legislative bijuralism constituted an additional challenge for the drafter who, fortunately, can rely on comparative law experts, as well as the sound advice of the jurilinguists, to produce legislative texts that are consistent with the principles of clarity in drafting legal documents.
\end{abstract}

Keywords Equal authority of the two official languages - Co-drafting · Jurilinguistics · Legislative bijuralism - Interpretation Act

Résumé L'avènement et le rôle grandissant de la jurilinguistique au sein de la filière législative fédérale sont étroitement liés à la reconnaissance graduelle de l'égale autorité des deux versions linguistiques des lois fédérales, ainsi qu'à la mise en place de la corédaction comme méthode rédactionnelle pouvant le mieux répondre aux exigences de l'égale autorité du français et de l'anglais législatifs. La jurilinguistique s'est graduellement instaurée au fédéral à compter du milieu des

L. A. Levert ( $\square)$

Ministère de la Justice du Canada, Ottawa, Canada

e-mail: 1llevert@yahoo.ca 
années 1970 et a rapidement donné lieu à la mise sur pied d'un groupe de jurilinguistique française composé de légistes francophones et de jurilinguistes. Ceux-ci ont joué un rôle majeur dans l'établissement de normes rédactionnelles permettant de rédiger la version française des lois conformément au génie qui lui est propre. Vers la fin des années 1990, des jurilinguistes anglophones furent également embauchés pour assurer la meilleure qualité linguistique possible de la version anglaise des lois fédérales et, de concert avec leurs collègues francophones, pour assurer l'équivalence fonctionnelle des deux versions linguistiques. La mise en place de la politique ministérielle sur le bijuridisme législatif constitua un défi additionnel pour le légiste, qui peut heureusement compter sur l'appui de spécialistes en droit comparé ainsi que sur les judicieux conseils des jurilinguistes pour néanmoins réaliser un texte de loi conforme aux impératifs de la lisibilité.

Mots clés Égale autorité des deux langues officielles · Corédaction · Jurilinguistique · Bijuridisme législatif · Loi d'interprétation

La jurilinguistique n'a pas toujours eu droit de cité au sein de la filière législative fédérale. Son avènement plutôt tardif et le rôle grandissant-et maintenant indispensable_qu'on lui a accordé sont directement tributaires de l'évolution du statut juridique de la version française des textes de loi fédéraux. Jusqu'en 1978, la jurilinguistique était, à toutes fins pratiques, absente du processus de rédaction des lois fédérales, ce qui semble difficile à croire vu la place importante qu'elle y occupe maintenant. Ce n'est que vers le milieu des années 1970 que les services d'un premier jurilinguiste (Alexandre Covacs), puis d'un second (Jean Kerby), furent retenus pour conseiller les légistes francophones; ces deux jurilinguistes leur proposaient notamment des modèles pour la rédaction de certaines dispositions types. Mais à cette époque, la version française était toujours, pour l'essentiel, le résultat d'une simple traduction, comme cela avait été le cas depuis la confédération.

Le présent article portera notamment sur la reconnaissance graduelle de l'égale autorité du français et de l'anglais dans les institutions fédérales, de même que sur certains aspects juridiques et pratiques propres au français législatif en contexte fédéral. Il sera bien sûr également question de l'instauration progressive de la jurilinguistique au sein des services législatifs fédéraux et d'un certain nombre de considérations pratiques propres à son insertion dans la filière législative fédérale.

\section{Article 133 de la Loi constitutionnelle de 1867}

L'article 133 de la Loi constitutionnelle de $1867^{1}$ prévoit que le français et l'anglais sont les deux langues d'usage au Parlement du Canada. Il prévoit également que les lois doivent être imprimées et publiées dans ces deux langues, mais il ne prévoit aucunement que les lois doivent être déposées, débattues et adoptées en français et

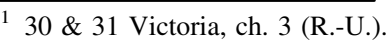


en anglais, et encore moins que ces deux langues jouissent d'un statut égal dans la législation fédérale. Ce sont les tribunaux qui se sont chargés d'extraire de cette disposition un certain nombre de principes fondamentaux. Puis sont venues s'ajouter la Loi sur les langues officielles de $1969^{2}$ et celle de $1988,{ }^{3}$ mais surtout la Charte canadienne des droits et libertés. ${ }^{4}$

\section{2 Égale autorité des deux versions}

En 1891, dans l'arrêt C.P.R. c. Robinson, ${ }^{5}$ la question se posa de savoir si les deux versions linguistiques du Code civil du Bas-Canada faisaient également autorité. La Cour suprême, constatant que les deux versions avaient toutes deux été soumises à la législature, puis avaient été adoptées et sanctionnées simultanément, en conclut que les deux versions linguistiques devaient logiquement avoir égale autorité. Ce n'est qu'en $1935^{6}$ que la Cour suprême eut l'occasion de répondre à la même question, mais cette fois par rapport aux deux versions linguistiques d'une loi fédérale. S'inspirant de l'arrêt C.P.R. c. Robinson, la Cour estima que les versions française et anglaise des lois fédérales ne pouvaient qu'avoir la même valeur juridique.

La Loi sur les langues officielles de $1969^{7}$ a reconnu l'égale autorité des deux versions linguistiques des lois fédérales de façon explicite lorsque, dans son paragraphe 9(1), le législateur a précisé que ces deux versions «font également foi». Mais il ne s'agissait là que d'une consécration législative. En $1979^{8}$ et à nouveau en $1985,{ }^{9}$ la Cour suprême du Canada a implicitement constitutionnalisé cette reconnaissance de l'égalité de statut du français et de l'anglais dans les textes de loi fédéraux. La constitutionnalisation formelle de l'égale autorité des deux versions a dû attendre l'avènement de la Charte canadienne des droits et libertés. ${ }^{10}$ Le paragraphe 18(1) de la Charte précise que les lois sont imprimées et publiées «en français et en anglais, les deux versions des lois ayant également force de loi».

\section{3 Égale autorité, mais traitement inégal de 1867 à 1978}

Malheureusement, «égale autorité» des deux versions n'a pas toujours été synonyme de «traitement égal» des deux versions en ce qui a trait à leur préparation et à leur

\footnotetext{
2 L.R.C. 1985, ch. 0-3.

3 L.R.C. 1985, ch. 31 (4 $4^{\mathrm{e}}$ suppl.).

${ }^{4}$ Loi constitutionnelle de 1982, édictée comme Annexe B de la Loi de 1982 sur le Canada, 1982, ch. 11 (R.-U.).

5 (1891) R.C.S. 292.

6 Dans l'arrêt R. C. Dubois, [1935] R.C.S. 378.

7 Précitée, note 2.

8 Blaikie c. Procureur général du Québec, [1979] 2 R.C.S. 1016.

9 Renvoi: Droits linguistiques au Manitoba, [1985] 1 R.C.S. 721.

10 Précitée, note 4.
} 
rédaction. En effet, de 1867 à 1978, la rédaction des lois fédérales se faisait exclusivement en anglais, la version française, quant à elle, résultant d'une simple traduction réalisée une fois complétée la version anglaise. En outre, il semble bien que les traducteurs disposaient de très peu d'information, en plus du texte à traduire, pour les appuyer dans leur travail. Ils étaient, pour l'essentiel, appelés à traduire des textes «en vase clos». Ils n'avaient, semble-t-il, accès ni aux documents pertinents du Cabinet, ni aux rédacteurs de la version anglaise. Faute d'information contextuelle adéquate, ils devaient donc, le plus souvent, s'en tenir à une traduction plutôt littérale, comme le faisaient les gens d'une autre génération lorsque, dans le cadre de leurs études classiques, ils étaient appelés à traduire vers le français des textes latins ou grecs dont le contexte leur échappait. Le moins que l'on puisse dire, c'est que les traducteurs des lois fédérales ne jouissaient pas de la latitude nécessaire pour formuler la version française selon le génie qui lui est propre. Bien que compétents, ils craignaient, vu le contexte d'alors, de s'éloigner du texte anglais. En outre, on leur imposait souvent des expressions calquées de l'anglais comme étant consacrées et donc irremplaçables.

Il est sans doute bon de rappeler que, depuis la fin des années 1940, la rédaction des projets de loi gouvernementaux et leur dépôt au Parlement doivent d'abord être autorisés par le Cabinet (c'est-à-dire le Conseil des ministres). La décision d'aller de l'avant avec un projet de loi est normalement précédée d'une note au Cabinet qui fait état de la problématique nécessitant l'intervention du Parlement, des diverses solutions possibles, de la solution privilégiée par le ministre responsable, et qui comprend normalement des instructions rédactionnelles portant sur le contenu de la mesure législative envisagée. La décision du Cabinet reprend, pour l'essentiel, le contenu de la note sur laquelle s'est basé le Cabinet pour en venir à sa décision. Il est donc très utile, voire nécessaire, pour le rédacteur d'avoir accès à cette documentation. Or avant 1978, il semble que seul le rédacteur de la version anglaise avait accès à ces documents, le traducteur devant se fier le plus souvent au texte anglais pris hors contexte et sans accès, semble-t-il, au rédacteur anglophone auprès de qui il aurait pu espérer aller chercher un supplément d'information.

\section{4 Étude spéciale du Commissaire aux langues officielles (1976)}

Cette réalité n'a bien sûr pas échappé aux francophones à travers le pays, et elle n'a surtout pas échappé à l'attention du Commissaire aux langues officielles, chargé, au titre de la Loi sur les langues officielles, de veiller au respect des deux langues officielles dans les institutions fédérales. À cet égard, dans une étude spéciale réalisée en décembre 1976 et portant sur le ministère de la Justice, le Commissaire invite le ministère de la Justice fédéral, responsable de la rédaction de l'ensemble des lois fédérales depuis 1948, à mettre en œuvre «un plan visant à assurer des droits et des privilèges égaux aux deux langues officielles, dans la rédaction des lois et l'examen des règlements, tout en respectant le génie de chacune des langues et les concepts des deux systèmes 
juridiques du pays». ${ }^{11}$ Bref, le ministère de la Justice fédéral est invité à accorder un traitement égal aux deux versions linguistiques dans la rédaction des lois et l'examen des règlements fédéraux.

\section{Mise sur pied d'un groupe de travail chargé du dossier}

Dans la foulée de cette étude, le ministère de la Justice s'est empressé de mettre sur pied un groupe de travail sous la présidence de Me Alban Garon, ancien juge en chef de la Cour canadienne de l'impôt, qui était alors haut fonctionnaire au ministère de la Justice avant de devenir directeur du programme français de rédaction législative à la Faculté de droit de l'Université d'Ottawa. Le groupe de travail, dont le mandat portait sur la rédaction des lois, était chargé de proposer des solutions susceptibles de remédier à la situation déplorée par le Commissaire aux langues officielles. Quelques mois plus tard, un second groupe de travail présidé par Me Alice Desjardins, alors avocate au ministère de la Justice et devenue par la suite juge à la Cour d'appel fédérale, fut mis sur pied pour examiner la situation en ce qui a trait à la rédaction et à l'examen des règlements. Le groupe de travail Garon a examiné de près comment d'autres pays, notamment la Belgique, la Suisse et la Communauté européenne, s'y étaient pris pour relever le défi de la rédaction législative bilingue ou multilingue.

Aucun des modèles examinés ne semblant répondre aux besoins particuliers du Canada, le groupe a donc recommandé au ministère de la Justice d'élaborer une méthode sui generis de rédaction qui assurerait l'égalité de statut des deux langues officielles, notamment la corédaction. Le rapport du groupe contenait également plusieurs recommandations portant sur la réorganisation des structures administratives de la Section de la législation du ministère de la Justice en vue de favoriser l'égalité de traitement des deux langues. ${ }^{12}$

\section{Traitement des deux versions linguistiques à compter de 1978}

Dans la foulée des recommandations formulées par le groupe de travail Garon, la Section de la législation du ministère de la Justice du Canada a procédé à l'inventaire des différentes méthodes envisageables (corédaction, rédaction

\footnotetext{
11 Recommandation 32 de l'étude spéciale en question, citée par Me Réjean Patry dans son ouvrage intitulé La législation linguistique fédérale et publié dans Documentation du Conseil de la langue française, et dont on peut retrouver le texte à l'adresse suivante: http://www.cslf.gouv.qc.ca/bibliothequevirtuelle/publication-html/?tx_iggcpplus_pi4\%5Bfile\%5D=publications\%2Fpubd106\%2Fd106ch3. html\#note122.

12 Voir la communication présentée par Me Gérard Bertrand, ancien premier conseiller législatif au ministère de la Justice du Canada, lors du séminaire national sur la rédaction et l'interprétation législatives, organisé sous l'égide de l'Institut canadien d'administration de la Justice du 19 au 21 août 1987, et intitulée Rédaction bilingue des lois.
} 
parallèle, rédaction alternée, rédaction partagée, et rédaction en partie double) ${ }^{13}$ et a d'ailleurs mis à l'essai, pendant un certain temps, la plupart de ces méthodes de rédaction visant à assurer l'égalité de traitement des deux versions officielles, y compris la rédaction des deux versions des projets de loi par le même rédacteur, méthode que l'on a appelée rédaction en partie double, terme emprunté à la comptabilité. La corédaction s'est rapidement imposée comme le choix le plus judicieux et le plus susceptible de répondre aux attentes exprimées par le Commissaire aux langues officielles dans son étude spéciale de 1976.

\section{La corédaction}

Le but premier de la corédaction_-personne ne s'en surprendra-est de veiller à ce que les deux versions linguistiques des lois fédérales reçoivent, au niveau de leur préparation, un traitement égal et à ce qu'elles soient toutes deux rédigées avec le même soin. Dès 1978, elle a été considérée comme la meilleure voie à suivre afin de remplacer le système qui était en place depuis la confédération et dont le résultat pervers était de subordonner l'une des deux versions (en l'occurrence la version française) à la seconde. La méthode rédaction-traduction (c.à-d. rédaction en anglais exclusivement, puis traduction vers le français) a donc été mise au rancart pour de bon en 1978. La corédaction législative permet de produire, à partir de documents du Cabinet rédigés dans les deux langues et d'instructions également fournies le plus souvent dans les deux langues par le ministère responsable de la mesure législative envisagée, deux versions originales et authentiques correspondant chacune au génie qui lui est propre.

En contexte de corédaction, chaque dossier législatif est assigné simultanément à deux rédacteurs législatifs (également appelés légistes) de la Section de la législation: un francophone, normalement formé en droit civil, et un anglophone, normalement formé en common law. «Après avoir établi un plan commun, les deux juristes rédigent leurs articles successifs, en en confrontant au fur et à mesure le sens et en en modifiant au besoin le plan». ${ }^{14}$ L'un des deux rédacteurs (parfois appelé premier rédacteur) a la responsabilité première du dossier, mais contrairement à ce qui se passait en contexte de traduction, le texte de départ n'est jamais immuable, le second rédacteur pouvant amener le premier rédacteur à modifier et à améliorer son texte. Il est important de noter que la responsabilité première du dossier est partagée à part égale entre légistes francophones et légistes anglophones. La coordination des aspects pratiques du dossier, notamment l'organisation des rencontres avec les chargés de projet du ministère client, relève normalement du premier rédacteur au dossier. Chaque rédacteur a la responsabilité de s'assurer, non seulement que son texte correspond bien à la décision du Cabinet et aux attentes du ministère client,

\footnotetext{
13 Ces diverses méthodes sont décrites en détail aux pages 25 à 28 d'un texte rédigé par Alexandre Covacs et intitulé La réalisation de la version française des lois fédérales du Canada, et présenté au IIIe Colloque international sur la rédaction des lois, tenu en septembre 1980 à Pointe-au-Pic (Québec), texte que l'on peut retrouver à l'adresse suivante: http://www.cslf.gouv.qc.ca/bibliotheque-virtuelle/ publication-html/?tx_iggcpplus_pi4\%5bfile\%5d=publications/pubf104/f104p1ch2.html.
}

14 Id., p. 25. 
mais également que les deux versions comportent le même message et auront ultimement le même effet juridique. Les jurilinguistes, dont il sera question cidessous, ont un rôle complémentaire important à jouer à cet égard.

On a souvent, à tort à mon avis, parlé de rédaction parallèle pour décrire le processus de corédaction. De nos jours, toutefois, comme la plupart des projets de loi sont rédigés simultanément en salle de corédaction, en la présence des chargés de projet, il serait sans doute de bon aloi de parler de rédaction parallèle pour décrire ce processus.

\section{Directive du Cabinet sur l'activité législative}

Dans sa directive intitulée Directive $d u$ Cabinet sur l'activité législative, ${ }^{15}$ le Cabinet «expose les attentes des ministres au sujet du processus d'élaboration des lois et règlements fédéraux et oriente de façon générale les activités des fonctionnaires à cet égard». Dans ce contexte, la directive rappelle l'importance que revêt, au sein de l'appareil gouvernemental, l'élaboration des règles de droit, et fait état d'un certain nombre de principes devant guider l'exercice de cette activité.

On y rappelle notamment l'importance de veiller à ce que la rédaction des textes de loi se fasse «dans le respect des deux langues officielles». Et à cet égard, la directive rappelle au lecteur que, parce que les lois fédérales doivent être adoptées dans les deux langues officielles en vertu de la Loi constitutionnelle de 1867 et que les deux versions font également foi, il est «de la plus haute importance que les projets de loi et de règlement soient préparés dans les deux langues officielles. L'une des versions ne saurait donc être une simple traduction de l'autre.» En conséquence, de poursuivre la directive, les autorités gouvernementales qui parrainent ces mesures «doivent s'assurer qu'elles sont en mesure d'élaborer les orientations et objectifs, de consulter et de donner des instructions aux légistes dans les deux langues officielles.» Le texte de la directive précise, enfin, que les deux versions linguistiques de la mesure législative envisagée doivent comporter le même message et produire le même effet.

La directive précise également que les deux versions doivent être établies de manière contemporaine. Conséquemment, le ministère responsable doit être en mesure de fournir des instructions aux rédacteurs et de répondre à leurs questions de fond, dans les deux langues officielles; il doit également être en mesure d'analyser et de critiquer les deux versions linguistiques. On rappelle également, dans la directive, que les deux textes doivent «revêtir la même qualité substantielle» et que, par conséquent, il est inacceptable que l'on se contente d'assurer la qualité substantielle d'une seule version (en l'occurrence, la version anglaise) sans trop s'attarder à l'autre version.

\footnotetext{
15 Lois et règlements: l'essentiel - Directive du Cabinet sur l'activité législative, document que l'on peut retrouver à l'adresse suivante: http://www.pco-bcp.gc.ca/index.asp?lang=fra\&page=information\&sub= publications\&doc=legislation/cabdir-dircab-fra.htm. Cette directive a remplacé la directive précédente, intitulée La filière législative et approuvée par le Cabinet le 16 avril 1981.
} 


\section{Arrivée d'un premier jurilinguiste et mise sur pied du Groupe de jurilinguistique française}

Retournons maintenant un peu dans le temps. Jusqu'en 1978, tel que cela a été évoqué ci-dessus, les lois fédérales étaient rédigées en anglais seulement, puis traduites, souvent littéralement, vers le français, de manière, espérait-on, à respecter à tout le moins la lettre de l'article 133 de la Loi constitutionnelle de 1867. La notion de légiste francophone était pour ainsi dire inexistante avant 1978. De plus, est-il besoin de le rappeler, les textes français fédéraux étaient, comme ils le sont toujours, réalisés dans un contexte bien particulier, soit celui d'une administration publique nettement dominée par la langue anglaise, dans un pays et un continent très majoritairement anglophones. Comment alors pourrait-on arriver à mettre en place un système de corédaction dont l'objectif premier serait la réalisation de deux versions originales et authentiques? Comment réaliser une version française autonome et de qualité dans ces circonstances? Le défi était de taille. Il était clair que, dorénavant, le style souhaité pour la rédaction de la version française allait devoir s'éloigner considérablement des traductions littérales auxquelles on avait habitué les Canadiens d'expression française pendant si longtemps, mais comment cela pourrait-il se faire?

La Section de la législation du ministère de la Justice comptait déjà quelques juristes francophones qui ne demandaient pas mieux que de devenir des légistes au même titre que les légistes anglophones qui, jusque-là, avaient eu l'exclusivité de la rédaction des lois fédérales. Le rôle de ces quelques juristes francophones consistait à réviser les traductions françaises qui étaient préparées par le Secrétariat d'État (plus précisément par le Bureau des traductions, devenu par la suite Bureau de la traduction dans les Lois révisées du Canada de 1985) afin d'en assurer la qualité au plan juridique. Leur travail était par la suite révisé par un spécialiste de la langue française du nom d'Alexandre Covacs, qui serait épaulé sous peu par un autre spécialiste du nom de Jean Kerby, qui était docteur en droit. Ces deux spécialistes n'étaient pas encore désignés sous le nom de jurilinguistes. Le terme jurilinguiste n'existait pas encore à l'époque, mais il ne tarderait pas à faire son apparition, à l'initiative d'ailleurs d'Alexandre Covacs à qui l'on en attribue la paternité. ${ }^{16}$ L'expression juriste linguiste existait déjà, mais elle s'appliquait à des juristes qui étaient également linguistes, sans pouvoir s'appliquer à des non-juristes. D'après les explications fournies à l'auteur du présent article par Alexandre Covacs, lors d'un entretien téléphonique, le 28 novembre 2012, le terme jurilinguiste fut créé pour désigner des personnes qui, sans être nécessairement juristes, sont des linguistes spécialisés dans la langue du droit.

Messieurs Covacs et Kerby formaient, avec les juristes francophones de la Section de la législation, le Groupe de jurilinguistique française qui, pendant de nombreuses années, s'est réuni périodiquement pour discuter de diverses questions

\footnotetext{
$\overline{16}$ Voir le mot jurilinguiste de même que le mot jurilinguistique dans la banque de données terminologiques et linguistiques Termium Plus du Bureau de la traduction, que l'on peut retrouver à l'adresse suivante: http://termiumplus.gc.ca/tpv2alpha/alpha-eng.html?lang=fra.
} 
relatives au français législatif-notamment pour approuver certaines dispositions types_et qui allait produire le Guide canadien de rédaction législative française. ${ }^{17}$

En 1978, tous les ingrédients étaient réunis pour permettre à la Section de la législation de passer de la traduction à la corédaction. On a donc graduellement éliminé la traduction, et les juristes francophones, encouragés par Messieurs Covacs et Kerby, ont commencé à rédiger eux-mêmes la version française des projets de loi plutôt que de simplement réviser les traductions pour en assurer la qualité au plan juridique. Les jurilinguistes revoyaient ensuite les textes rédigés par les légistes francophones pour en assurer la qualité linguistique. Bien sûr, la corédaction s'est implantée graduellement et il a fallu embaucher plusieurs légistes francophones avant d'être en mesure de corédiger l'ensemble des projets de loi gouvernementaux. La mise en œuvre graduelle de la corédaction a fourni aux deux premiers jurilinguistes de la Section de la législation l'occasion de travailler étroitement avec les légistes francophones de l'époque afin de jeter les bases d'un style authentiquement français qui perdure depuis.

\section{La révision des lois de 1985}

Au début des années 1980, le ministère de la Justice fédéral entreprend une sixième révision des lois fédérales. C'est alors qu'un troisième jurilinguiste (Bernard Méchin) se joint aux légistes du ministère, plus particulièrement à l'équipe chargée de la révision des lois de 1985, afin d'appuyer les travaux de la Commission de révision des lois qui portaient, dans une large mesure, sur l'amélioration de la version française des lois fédérales. Cela signifiait en pratique que la Commission avait pour mandat de remanier la version française des lois existantes, surtout les plus anciennes, en s'inspirant des normes rédactionnelles établies par la Section de la législation depuis l'avènement de la corédaction. Ces nouvelles normes applicables à la rédaction de la version française des projets de loi étaient d'abord et avant tout l'œuvre des jurilinguistes et du Groupe de jurilinguistique française. La Commission avait donc elle aussi besoin d'un jurilinguiste qui puisse la guider dans cette tâche. Le nouveau jurilinguiste se devait de travailler en étroite collaboration avec les jurilinguistes de la Section de la législation et le Groupe de jurilinguistique française, et il n'y manqua pas. Une fois les travaux de révision complétés, il s'est joint à la Section de la législation pour faire équipe avec Alexandre Covacs et dispenser ses judicieux conseils aux légistes chargés de la rédaction des projets de loi gouvernementaux. Jean Kerby était malheureusement décédé entre-temps.

\section{Trois pionniers}

Messieurs Covacs, Kerby et Méchin ont joué un rôle majeur dans la création du style qui prévaut maintenant pour la rédaction de la version française des lois

\footnotetext{
$\overline{17}$ On peut retrouver cette publication à l'adresse suivante: http://publications.gc.ca/site/fra/33607/ publication.html.
} 
fédérales. Bien sûr, ils n'ont pas tout inventé. Ils se sont inspirés des modèles existant au Québec, en France et ailleurs, tout en prenant soin, en concertation avec les légistes, de ne pas importer dans la législation fédérale des expressions, des modèles ou des tournures qui auraient pu poser problème, notamment au plan juridique. Plus souvent qu'autrement, ils ont dû créer de toutes pièces de nouveaux modèles correspondant au contexte particulier de la législation fédérale. C'est sans doute au niveau de la terminologie que leur rôle a été le plus marquant, comme c'est d'ailleurs encore le cas pour les jurilinguistes du ministère de la Justice fédéral qui ont pris la relève des trois pionniers des premières années de la corédaction. Est-il besoin de mentionner que la terminologie française proposée dans les instructions fournies aux rédacteurs francophones par les chargés de projet des ministères clients laisse souvent à désirer et nécessite souvent des recherches plus approfondies.

Les jurilinguistes de la première heure ont joué un rôle d'encadrement absolument essentiel auprès des légistes francophones qui ont, les premiers, relevé le défi de la corédaction. En effet, en 1978, la transition de la traduction souvent littérale à la rédaction de textes français originaux et conformes au génie de la langue ne pouvait s'effectuer sans heurt. Les légistes francophones embauchés par la Section de la législation étaient d'abord et avant tout recrutés pour leurs compétences juridiques. Bien sûr, les gens chargés de recruter les rédacteurs faisaient —et le font toujours-de grands efforts pour embaucher des juristes possédant également une bonne maîtrise de la langue française. Mais ces recrues n'étaient tout de même pas des experts de la langue française. En principe, ces juristes avaient une bonne connaissance de la langue, mais ils ne maîtrisaient pas toutes les subtilités propres au génie de la langue, comme c'était le cas pour les jurilinguistes. Compte tenu du contexte particulier dans lequel on rédige les textes français au fédéral, le recours à des experts de la langue française en contexte juridique s'imposait sans contredit.

\section{Des jurilinguistes anglophones?}

Les rédacteurs anglophones étaient-ils davantage des experts de la langue anglaise que les rédacteurs francophones pouvaient l'être de la langue française? Pas du tout, mais les choses étaient quand même fort différentes dans leur cas. En effet, ils pouvaient s'inspirer de modèles existant ailleurs au pays, au Royaume-Uni et dans bien d'autres pays anglophones. De plus, les ministères responsables des textes de loi à rédiger ont toujours été en mesure de fournir aux rédacteurs anglophones la terminologie appropriée au domaine couvert par la législation envisagée. Et de toute manière, au début de la corédaction, les rédacteurs de langue anglaise avaient en général beaucoup plus d'expérience comme légistes que l'équipe francophone dont la plupart des membres étaient de nouvelles recrues. En outre, les rédacteurs anglophones pouvaient compter sur l'appui d'une équipe de réviseurs rédactionnels chevronnés qui pouvaient généralement les conseiller sur les questions d'ordre linguistique et pouvaient donc, dans une certaine mesure, agir à titre de jurilinguistes anglophones. Le rôle des réviseurs rédactions sera d'ailleurs abordé plus spécifiquement ci-dessous. 
Cependant, au fil du temps, plusieurs rédacteurs anglophones ont réalisé jusqu'à quel point les conseils des jurilinguistes permettaient d'améliorer la qualité de la version française et parfois même, indirectement, de la version anglaise. Après tout, eux aussi, tout comme leurs collègues francophones, étaient d'abord et avant tout des juristes, sans spécialité particulière en matière linguistique. Ils ont donc commencé à demander à la direction de leur fournir des jurilinguistes anglophones. Vers la fin des années 1990, le premier conseiller législatif d'alors, en l'occurrence l'auteur de ce texte, créa officiellement une équipe de jurilinguistes. Celle-ci fut placée sous la responsabilité d'un des rédacteurs francophones, Me André Labelle qui est présentement à l'emploi de la Cour internationale de justice, à La Haye. Me Labelle, qui est également linguiste, avait appuyé cette initiative. Il fut alors décidé que cette nouvelle équipe de jurilinguistes comprendrait à la fois des jurilinguistes francophones et anglophones. Les membres anglophones de l'équipe étaient, et sont toujours, moins nombreux que leurs collègues francophones puisque les besoins en matière de jurilinguistique sont moins importants en anglais qu'en français, en particulier en ce qui a trait à la terminologie.

\section{Rôle actuel des jurilinguistes au fédéral}

La création d'une équipe de jurilinguistes francophones et anglophones a amené les autorités à modifier quelque peu le rôle des jurilinguistes. Le rôle des tout premiers jurilinguistes consistait exclusivement à assurer la qualité verticale de la version française, c'est-à-dire la qualité intrinsèque de la version française. Lors de la création d'une équipe de jurilinguistes «bilingue» à la fin des années 1990, on confia à cette équipe une tâche additionnelle, soit celle d'assurer l'équivalence horizontale des textes de loi. Dorénavant, les jurilinguistes auraient donc un double rôle, soit celui d'assurer la qualité de la langue (française ou anglaise, selon le cas) ainsi que celui d'assurer l'équivalence fonctionnelle des deux versions linguistiques. Cela dit, il n'est pas inutile de rappeler que la responsabilité d'assurer l'équivalence des deux versions d'un projet de loi revient d'abord et avant tout aux corédacteurs responsables du dossier, ainsi qu'aux représentants du ministère client. Cependant, on demande également aux jurilinguistes d'exercer ce rôle puisqu'ils peuvent le faire de façon plus objective et avec plus de recul que les corédacteurs ou les chargés de projet du ministère client.

Le rôle actuel des jurilinguistes peut donc se résumer ainsi: étant des spécialistes de la langue juridique, ils ont pour mandat d'aider les rédacteurs à atteindre le meilleur niveau de langue possible dans la réalisation des lois et des règlements fédéraux. Ils veillent à ce que le style, la terminologie et la formulation employés soient appropriés à la rédaction des lois et des règlements, aux sujets traités ainsi qu'aux auditoires visés. Enfin, ils sont chargés de relever toute contradiction ou contresens entre les deux versions et de les porter à l'attention des deux rédacteurs à qui ils font part de leurs suggestions portant sur l'une ou l'autre version linguistique.

Voici comment Me André Labelle, qui a été le premier directeur de l'équipe de jurilinguistique bilingue mise sur pied au sein du ministère de la Justice fédéral à la fin des années 1990, décrit le rôle du jurilinguiste: 
Le jurilinguiste est un spécialiste de la langue juridique dont la mission fondamentale consiste à aider les légistes à exprimer la règle de droit de la meilleure façon possible. Il veille à la qualité linguistique des textes législatifs, notamment en ce qui a trait au style, à la terminologie et à la phraséologie propres à la rédaction législative et aux sujets traités, et assure la concordance des version française et anglaise des textes.

Le jurilinguiste prend part au processus d'élaboration du texte dès les premières étapes, notamment par les consultations ponctuelles ou les recherches documentaires qu'il effectue pour le légiste. Tenu en principe de réviser à fond le projet que lui confie celui-ci, il doit être apte à reformuler des pans entiers du texte, à en renouveler complètement la terminologie, à proposer au besoin une restructuration plus logique et à se faire du texte, même et surtout si celui-ci est très long, une vue d'ensemble qui lui permette d'attirer l'attention des responsables sur ses éventuels manques de cohérence. Il peut ainsi être amené à le récrire dans une proportion parfois considérable, sinon en totalité, de façon que les éléments terminologiques, stylistiques, structurels et logiques en soient conformes aux normes rédactionnelles propres à la fois au secteur du droit et aux différents domaines techniques visés, et ce tant sur le plan interne que sur le plan comparatif (concordance des deux versions). ${ }^{18}$

Il est important de noter que les recommandations des jurilinguistes n'ont pas force obligatoire; les légistes restent maîtres de leur dossier. Cependant, les avis exprimés par les jurilinguistes sont généralement pris très au sérieux par les légistes.

Comme pour les rédacteurs législatifs, le travail des jurilinguistes s'effectue souvent dans des conditions difficiles, notamment en ce qui a trait aux délais dont ils disposent pour effectuer leur travail. Les délais qui leur sont impartis sont souvent nettement trop courts pour leur permettre d'effectuer un travail en profondeur, notamment en ce qui a trait aux recherches terminologiques qui s'imposent. Il arrive même que certains textes ne soient pas soumis à leur examen, faute de temps. Depuis toujours, les délais accordés pour la rédaction des textes de loi sont trop courts. Depuis quelques années, la situation a nettement empiré, surtout avec l'avènement des salles de corédaction où les projets de loi sont rédigés en la présence des chargés de projet du ministère client, mais sans la présence des jurilinguistes, qui sont consultés par la suite, dans la mesure où le temps le permet.

\section{Distinction entre le rôle de jurilinguiste et celui de réviseur rédactionnel}

Les légistes fédéraux peuvent compter non seulement sur l'appui d'un groupe de jurilinguistes, mais également sur celui d'un groupe de réviseurs rédactionnels. D'aucuns se demandent si les deux groupes sont vraiment nécessaires. Ne font-ils

\footnotetext{
18 Extrait tiré de la p. 6 d'une communication présentée par Me André Labelle lors d'un colloque sur la traduction juridique tenu à Genève, en février 2000; on peut retrouver le texte (La corédaction des lois fédérales au Canada_Vingt ans après: quelques réflexions) à l'adresse suivante: http://www.tradulex. com/Actes2000/LABELLE.pdf.
} 
pas double emploi? Un bon réviseur rédactionnel ne peut-il pas également jouer le rôle de jurilinguiste? La réponse est non et voici pourquoi.

Voyons d'abord ce qu'est le rôle des réviseurs rédactionnels. Pour l'essentiel, leur rôle est de veiller à ce que les idées contenues dans les textes de loi soient formulées clairement et logiquement, dans une langue grammaticalement correcte, d'assurer le respect des conventions rédactionnelles et des normes régissant la présentation et le style des textes de loi, de même que des modèles existants, et de veiller à ce que les textes soient exempts de fautes d'orthographe. Ils sont notamment chargés de conseiller les jeunes légistes quant aux techniques rédactionnelles à utiliser. Les légistes comptent sur eux pour la vérification des renvois de même que pour assurer l'exactitude des annotations historiques contenues dans les textes de loi. Ils conseillent les légistes sur ce qui devrait se retrouver dans des annexes à la fin des textes de loi et sur la façon de présenter ces annexes. Ils surveillent la cohérence dans l'utilisation des termes choisis par le légiste, surtout lorsque ceux-ci sont définis. À Ottawa, les réviseurs rédactionnels ont également la responsabilité de publier les lois et de tenir à jour les banques de données des lois et des règlements. Ils tiennent également à jour divers tableaux tels le tableau des entrées en vigueur et le tableau des lois d'intérêt privé.

Leur travail peut à l'occasion, mais si peu, faire double emploi avec celui des jurilinguistes lorsqu'il s'agit d'assurer l'exactitude grammaticale et orthographique des textes de loi. Mais, comme nous l'avons vu ci-dessus, le rôle des jurilinguistes va beaucoup plus loin à cet égard. En effet, ceux-ci peuvent suggérer une reformulation complète de certaines dispositions proposées de manière à mieux respecter le génie de la langue. Ils sont également chargés d'effectuer des recherches parfois très poussées sur des questions terminologiques. De plus, comme nous l'avons déjà mentionné, ils sont chargés, de concert avec les légistes et les chargés de projet du ministère client, de veiller à ce que les deux versions linguistiques aient le même sens et le même effet.

Les deux disciplines (jurilinguistique et révision rédactionnelle) sont donc complémentaires et sont toutes deux nécessaires pour assurer la meilleure qualité possible des textes de loi.

\section{Politique sur le bijuridisme législatif (impact sur la langue du droit)}

En 1995, le ministère de la Justice du Canada s'est doté d'une politique en matière de bijuridisme législatif. Cette politique existait déjà depuis un certain temps au sein et aux fins de la Direction des services législatifs, mais en 1995, elle est officiellement devenue une politique ministérielle. ${ }^{19}$ La politique en question est le résultat direct de consultations menées par la Direction des services législatifs, vers la fin des années 1980 et le début des années 1990, afin de vérifier et de valider le degré de satisfaction des lecteurs de la version française des lois fédérales relativement au nouveau style mis en place après l'avènement de la corédaction en

\footnotetext{
$\overline{19}$ On peut retrouver cette politique à l'adresse suivante: http://www.justice.gc.ca/fra/bijurilex/politiquepolicy.html.
} 
1978. Malheureusement, aucun rapport officiel n'a été produit à la suite de cette consultation, mais l'auteur du présent article, qui a mené ces consultations en compagnie de la sous-ministre déléguée de l'époque, l'honorable Anne-Marie Trahan, se souvient très bien des constats que l'initiative a permis de faire.

La plupart des intervenants ont trouvé que la qualité de la version française des textes réalisés depuis 1978 était nettement meilleure qu'auparavant. Certains nous ont reproché de parfois utiliser des termes ou de recourir à des formulations un peu trop recherchés, mais pour l'essentiel, la nouvelle façon de rédiger le texte français des lois fédérales a reçu l'appui enthousiaste des gens consultés, avec une réserve importante toutefois. On nous a en effet reproché de ne pas suffisamment tenir compte des deux systèmes de droit privé dans chacune des deux versions linguistiques des textes fédéraux. Certaines de ces consultations ont été menées dans le cadre des rencontres régulières du Programme national d'administration de la justice dans les deux langues officielles (mieux connu sous son acronyme PAJLO). Le PAJLO était un programme fédéral (géré par le ministère de la Justice fédéral en partenariat avec Patrimoine Canada et, dans une moindre mesure, le Bureau de la traduction) visant à faciliter l'accès à la justice dans les deux langues officielles, notamment par le financement d'ouvrages et d'outils de toutes sortes susceptibles d'appuyer l'atteinte de cet objectif. L'auteur du présent texte a présidé le PAJLO pendant plusieurs années. Les consultations tenues dans le cadre des rencontres du PAJLO avaient l'immense avantage de nous permettre d'entendre le point de vue de représentants des quatre auditoires juridiques du pays. Ainsi on nous a fait valoir que les anglophones du Québec devaient souvent aller lire la version française du texte consulté pour être en mesure de bien comprendre les concepts et les notions juridiques évoqués dans le texte. De la même manière, des francophones venant de l'extérieur du Québec nous ont fait valoir qu'ils devaient souvent aller lire la version anglaise des textes de loi fédéraux afin de mieux en saisir la portée et l'effet juridique souhaité.

Trop souvent, en effet, la version française des textes de loi fédéraux était jusquelà rédigée d'abord et avant tout en fonction du droit civil, alors que la version anglaise reflétait, pour l'essentiel, la common law. Les enjeux du bijuridisme législatif avaient très peu retenu l'attention des autorités avant la tenue de ces consultations. Il est vrai que la question s'était posée dans le cadre des travaux portant sur la révision des lois de 1985 et que l'auteur de ce texte a dirigés de 1981 à 1988, mais elle avait été réglée plutôt sommairement. Les juristes chargés de réviser les lois fédérales avaient, en effet, constaté que, dans certaines dispositions touchant au droit privé, les légistes avaient parfois eu recours à la fois à la terminologie du droit civil et à celle de la common law. On s'était alors posé la question de savoir s'il fallait généraliser cette approche. Malheureusement, il fut décidé qu'il n'appartenait pas à la Commission de révision des lois ${ }^{20}$ d'effectuer pareil virage dans le cadre de la révision en cours et que, de toute manière, les quelques dispositions où l'on avait eu recours aux notions et termes propres aux deux systèmes de droit du pays étaient

\footnotetext{
${ }^{20}$ Organisme créé par la Loi sur la révision et la codification des textes législatifs (L.R.C. 1985, ch. S-20).
} 
exceptionnels et sans doute dus à une situation particulière. Bref, il n'était pas question de généraliser cette pratique.

La décision prise par la Commission de révision des lois était fondée sur l'existence d'une disposition contenue dans la première Loi sur les langues officielles $^{21}$ et qui n'avait pas été reprise dans la seconde Loi sur les langues officielles. ${ }^{22}$ L'alinéa 9 (2) c) de la loi de 1969 prévoyait en effet que, «si les notions ou réalités qu'énonce le texte ne sont compatibles avec le système juridique ou les institutions d'un lieu du Canada où il est destiné à s'appliquer que dans l'une de ses versions, c'est cette dernière qui s'applique en ce lieu». Sur la base de cette disposition, la Commission de révision des lois avait jugé que le fait qu'un seul des deux systèmes juridiques canadiens soit pris en compte dans l'une ou l'autre des deux versions linguistiques d'un texte de loi ne posait aucun problème au plan juridique. La Commission avait sans doute raison, mais la solution retenue était assez peu respectueuse des attentes des quatre auditoires juridiques canadiens.

C'est pourquoi, vers le milieu des années 1990, la Direction des services législatifs du ministère de la Justice du Canada, dans un premier temps, puis le ministère lui-même, dans un deuxième temps, ont adopté une politique portant sur le bijuridisme législatif. Pour l'essentiel, cette politique reconnaît formellement le droit des quatre auditoires juridiques canadiens de lire les lois dans la langue officielle de leur choix et d'y retrouver les concepts et notions juridiques correspondant au système juridique qui s'applique dans leur région du pays. Par cette politique, qui est toujours en place, le ministère de la Justice «s'engage, chaque fois qu'un projet de loi ou de règlement fédéral touche au droit privé provincial ou territorial, à rédiger chacune des deux versions de ce texte en tenant compte également de la terminologie, des concepts, des notions et des institutions propres aux deux régimes de droit privé canadiens». ${ }^{23}$

\section{6 Éléments contextuels favorisant la mise en place d'une telle politique}

La mise en place d'une telle politique aurait été totalement impensable, voire irresponsable, dix années plus tôt. Le contexte des années 1990 permettait au ministère de se doter d'une telle politique en sachant que les éléments essentiels à sa mise en œuvre étaient maintenant bien présents dans le paysage bijuridique canadien. Le préambule de la politique fait d'ailleurs état d'un certain nombre de ces éléments contextuels: la corédaction au fédéral (qui était en place depuis déjà une quinzaine d'années), la formation en droit bilingue et bijuridique offerte par certaines universités canadiennes, les outils terminologiques réalisés grâce à l'appui du PAJLO, l'avènement du nouveau Code civil du Québec qui avait entraîné la mise en place d'un projet d'harmonisation des lois fédérales avec les dispositions du nouveau Code.

\footnotetext{
21 Précitée, note 2.

22 Précitée, note 3.

23 Loc. cit., note 19.
} 
La politique visait à rendre davantage présents le droit civil dans la version anglaise, et la common law dans la version française. Augmenter la présence du droit civil dans la version anglaise représentait un moindre défi vu que la terminologie existait déjà dans la version anglaise du Code civil du Québec, de même que dans le Code civil du Bas-Canada. Évidemment, le Centre de recherche en droit privé et comparé du Québec de l'Université McGill, rebaptisé le Centre Paul-André Crépeau de droit privé et comparé du Québec, le 14 mars 2012, a facilité la tâche avec ses remarquables travaux en matière de droit privé, qui ont donné lieu à la publication de multiples lexiques et dictionnaires (français et anglais).

La situation en ce qui a trait à la common law était tout autre. Depuis toujours, la common law avait été pensée et écrite en anglais. Afin de répondre aux impératifs du bilinguisme de plus en plus présent, non seulement au sein des institutions fédérales, mais également dans les institutions provinciales, et en particulier devant les tribunaux, on a commencé à enseigner la common law en français aux universités de Moncton et d'Ottawa. On a également entrepris d'élaborer une terminologie de common law en français, notamment au Centre de traduction et de terminologie juridiques de l'Université de Moncton. Le PAJLO, dont il a été question ci-dessus, a mis en place un processus de normalisation du vocabulaire de common law, processus qui se poursuit toujours, tout en appuyant la réalisation de divers outils favorisant l'accès à la justice dans les deux langues officielles. Grâce à ces diverses initiatives, la rédaction véritablement bijuridique des lois fédérales devenait un objectif de plus en plus envisageable. Il est vrai que les lois fédérales avaient toujours été bijuridiques en ce sens qu'elles avaient toujours tenu compte du droit civil dans la version française et de la common law dans la version anglaise, mais les cas où le législateur fédéral avait tenu compte de ces deux systèmes dans chacune des deux versions linguistiques étaient rarissimes.

Il est important de souligner l'apport des jurilinguistes dans le dossier de la normalisation du vocabulaire français de common law. Les fiches documentaires destinées à alimenter les discussions des experts chargés de la normalisation étaient souvent préparées par des jurilinguistes d'expérience. Les gens qui siégeaient au sein du comité de normalisation prenaient bien soin de consulter des jurilinguistes quant à l'à-propos de telle ou telle recommandation avant de participer aux discussions devant mener à une décision de normalisation. Les impératifs d'ordre juridique ont parfois pris le dessus sur les impératifs d'ordre strictement linguistique, mais il n'en demeure pas moins que les jurilinguistes ont eu et ont toujours un rôle majeur à jouer dans l'établissement d'une terminologie française de common law.

\section{Spécialistes en droit comparé et jurilinguistes}

Le fait de mettre à la disposition de nos rédacteurs législatifs les divers outils terminologiques dont il a été question ci-dessus n'en faisait pas pour autant, comme par magie, des experts des deux systèmes de droit privé canadiens. Bien sûr, certains d'entre eux avaient étudié à la fois le droit civil et la common law, mais ce n'était 
certes pas le cas de la majorité de nos légistes. À cet égard, l'auteur de cet article, qui est également l'auteur de la politique sur le bijuridisme législatif, est prêt à reconnaitre que la mise en place de cette politique au niveau de la Direction des services législatifs a sans doute été faite quelque peu prématurément. Il aurait sans doute d'abord fallu offrir à nos rédacteurs un meilleur encadrement pour les guider à travers les méandres du bijuridisme législatif, mais il était impératif d'envoyer, dans les meilleurs délais, un signal clair aux quatre auditoires juridiques canadiens quant aux intentions du ministère de la Justice fédéral.

Cet encadrement est venu quelques années plus tard lorsque la Direction des services législatifs embaucha, à l'été 2000, une première spécialiste en droit comparé, en la personne de Me France Allard, de l'Université McGill. Avec le temps, plusieurs autres spécialistes sont venus s'ajouter à cette première recrue. Ces spécialistes, formés en droit civil et en common law, sont appelés à revoir les projets de loi pour s'assurer que les dispositions qui touchent au droit privé tiennent également compte des deux systèmes de droit du pays dans chacune des deux versions linguistiques. Ils sont donc appelés à conseiller les légistes sur cet aspect bien précis des projets de loi et de règlement. Les légistes demeurent toujours maitres de leurs dossiers, et ce sont eux qui prendront les décisions qui s'imposent, mais il va de soi que les rédacteurs ne prendront pas à la légère les conseils que leur prodiguent les experts en droit comparé.

Le rôle exercé par ces experts n'a pas pour effet d'écarter la participation des jurilinguistes en ce qui a trait aux dispositions dites «bijuridiques» des textes législatifs. Les conseils judicieux des jurilinguistes sont tout aussi importants, et sans doute plus importants, lorsqu'il s'agit de ce type particulier de dispositions législatives, puisque le bijuridisme législatif a le potentiel, si l'on n'y prend garde, d'alourdir les dispositions législatives. Pourtant ces dispositions, comme le reste du texte de loi en question, doivent être le plus claires possible et de la meilleure qualité linguistique possible. Légistes, experts en droit comparé et jurilinguistes ont donc la responsabilité conjointe de travailler à assurer la qualité «bijuridique» et linguistique des textes de loi, tout en étant bien conscients de l'importance de produire des textes dont la lisibilité répondra aux attentes du public cible.

\section{Jurilinguistique, bijuridisme législatif et lisibilité des textes législatifs: confrontation ou collaboration?}

Jurilinguistique, bijuridisme législatif et lisibilité des textes de loi poursuivent tous des objectifs fort louables, mais dans leur mise en œuvre, ces trois réalités peuvent parfois donner lieu à des tensions, voire des conflits, entre leurs tenants respectifs. Ainsi le bijuridisme législatif poussé à l'extrême permettra assurément de produire des textes de loi fort respectueux des quatre auditoires juridiques du pays, mais cela se fera sans doute au détriment de la qualité linguistique, voire de la lisibilité, des textes en question. $\mathrm{Si}$, par exemple, au nom des principes du bijuridisme législatif, les légistes tiennent, dans les deux versions (comme il se doit), à faire état systématiquement de la terminologie propre à chacun des deux grands systèmes de droit privé qui prévalent au Canada à chaque fois que le texte à rédiger touche au 
droit privé, le texte qui résultera de cet exercice risque d'être lourd et sans doute difficile à lire et à comprendre, et ce en contravention flagrante des principes de lisibilité et des exigences de haute qualité linguistique des textes de loi. Le recours à une terminologie et à une formulation plus neutres ou encore à des définitions consistant à définir un terme neutre ou générique (c'est-à-dire un terme qui ne relève d'aucun des deux systèmes de droit privé du pays) comme comportant les différentes notions de droit civil et de common law qu'il faut évoquer aux fins du texte de loi en question permet souvent d'alléger la formulation du texte. Cela ne veut pas dire que la technique qui consiste à faire état, dans les deux versions linguistiques, de la terminologie propre à chacun des deux systèmes de droit privé (doublet simple) ou qui consiste à faire état, dans deux alinéas distincts (doublet avec alinéas), du droit applicable au Québec, d'une part, et du droit applicable dans les provinces de common law, d'autre part, soit condamnable dans tous les cas. Il faut au contraire encourager le recours judicieux à la technique du doublet.

De la même manière, le jurilinguiste qui s'attarde uniquement à assurer la meilleure qualité linguistique du texte qu'on lui soumet, sans égard aux principes sous-jacents au bijuridisme législatif ou à la lisibilité, peut produire un texte qui est un bijou au plan linguistique, mais dont la formulation risque de respecter assez peu, voire pas du tout, les quatre auditoires juridiques du pays de même que les principes élémentaires de lisibilité. Il peut arriver à l'occasion qu'à force de vouloir produire un texte parfait au plan linguistique, on finisse par produire un texte qui ne soit pas facilement accessible à ses destinataires.

Il est donc de la plus haute importance que jurilinguistique, bijuridisme législatif et lisibilité fassent bon ménage et que l'on puisse, dans le travail de rédaction de tous les jours, en venir à un juste équilibre entre les trois. Ainsi, opter pour une terminologie et une formulation que tout le monde peut facilement comprendre plutôt que de recourir à des termes recherchés qui sont peu utilisés en contexte canadien ou dans le domaine de spécialité visé par la loi en question, constitue un choix judicieux, pourvu bien sûr que les termes et formulations retenus soient corrects au plan linguistique. Il ne s'agit pas de diminuer le niveau de la langue législative, mais de choisir, parmi des termes tout à fait corrects, ceux avec lesquels le lecteur éventuel est le plus familier.

\section{Article 8.2 de la Loi sur l'interprétation}

La décision prise par le ministère de la Justice de veiller à ce que tous les textes de loi touchant au droit privé tiennent également compte des deux systèmes de droit en vigueur au pays, et ce dans les deux versions linguistiques, a nécessité l'ajout d'une nouvelle disposition dans la Loi d'interprétation ${ }^{24}$ fédérale. Le but premier de cette nouvelle disposition (article 8.2) est de fournir une aide additionnelle à l'interprète des dispositions législatives fédérales de nature bijuridique, notamment en ce qui a trait aux dispositions où l'on a recours à la fois à des termes relevant respectivement du droit civil et de la common law, ou encore à des termes qui peuvent avoir un sens

${ }^{24}$ L.R.C. 1985 , ch. I-21. 
différent, selon qu'on les applique au Québec ou ailleurs au pays. La disposition en question se lit comme suit:

Sauf règle de droit s'y opposant, est entendu dans un sens compatible avec le système juridique de la province d'application le texte qui emploie à la fois des termes propres au droit civil de la province de Québec et des termes propres à la common law des autres provinces, ou qui emploie des termes qui ont un sens différent dans l'un et l'autre de ces systèmes.

Cette disposition, qui date de 2001, ne doit pas être vue comme un encouragement à recourir le plus souvent possible à la technique du doublet simple dont il a été question ci-dessus, au détriment de la méthode dite de la neutralité dont il a également été question ci-dessus. Le législateur reconnaît que, dans certaines circonstances, le bijuridisme législatif exige que l'on ait recours au doublet simple et, par conséquent, il a jugé utile de modifier la Loi d'interprétation par l'insertion d'une disposition susceptible de guider le lecteur qui est appelé à interpréter et à appliquer une disposition dans laquelle onù utilise la technique du doublet simple ou dans laquelle on utilise un terme qui a un sens différent en droit civil et en common law. Sans ce nouvel article 8.2, l'interprète du texte comportant un doublet simple pourrait se sentir obligé, en vertu d'une présomption interprétative, de donner un sens à tous les termes utilisés dans la disposition en question, qu'ils soient propres au système de droit civil ou au système de common law, où que l'on soit au pays. En effet, en matière d'interprétation législative, il existe une présomption voulant que le législateur ne parle pas pour ne rien dire. En vertu de cette présomption, on présume donc normalement que chaque terme utilisé dans la disposition que l'on cherche à interpréter et à appliquer a été retenu en vue de produire quelque effet, ${ }^{25}$ plutôt que de conclure que certains termes utilisés dans le texte sont tout simplement superflus.

Or l'article 8.2 permet justement à l'interprète d'une telle disposition de laisser de côté certains termes, en l'occurrence les termes propres à la common law, s'il s'agit d'appliquer le texte au Québec, ou encore les termes propres au droit civil, s'il s'agit d'appliquer le texte dans une province de common law. De la même manière, l'article 8.2 permet de donner un sens différent à un même terme (le terme fiducie par exemple), selon que l'on se trouve au Québec ou ailleurs au pays. Le rédacteur législatif n'a donc pas à s'inquiéter du fait qu'il utilise, dans un texte touchant au droit privé, des termes qui, en réalité, ne recevront d'application que dans certaines régions du pays.

\section{Conclusion}

L'article 133 de la Loi constitutionnelle de 1867 et l'interprétation que lui ont donnée les tribunaux ont eu un impact sur les exigences linguistiques applicables en matière de législation que l'on ne soupçonnait guère en 1867. Sans cet article de

\footnotetext{
25 Voir Pierre-André Côté (avec la collaboration de Stéphane Beaulac et Mathieu Devinat), Interprétation des lois, $4^{\mathrm{e}}$ édition, les éditions Thémis, 2009, à la page 318.
} 
notre constitution et sans la Charte canadienne des droits et libertés, la jurilinguistique n'aurait sans doute jamais vu le jour dans le contexte de la rédaction des lois fédérales. Et maintenant, il n'est pas exagéré de soutenir que la contribution des jurilinguistes au processus de rédaction des lois est essentielle pour assurer un traitement égal aux deux versions linguistiques, de même que pour assurer le plus haut niveau de qualité linguistique des textes législatifs.

Et pourtant, au départ, on aurait pu croire que leur rôle allait consister essentiellement à guider les rédacteurs francophones dans leurs premiers pas sur le chemin de la corédaction et que, une fois mis en place un bon guide de rédaction législative française, un légiste le moindrement débrouillard aurait pu se passer de l'appui des jurilinguistes. Mais leur apport, à l'époque de la transition de la traduction à la corédaction, a permis de constater que cette nouvelle discipline contribuait de façon significative à améliorer la qualité de la langue législative, à tel point que, quelques années plus tard, ce service fut également offert aux légistes anglophones.

On a tôt fait de conclure que la rédaction législative et la jurilinguistique sont deux disciplines distinctes, mais dont la complémentarité ne fait plus de doute. L'expérience des quelque trente-cinq dernières années a clairement démontré que la corédaction législative requiert les services à la fois des rédacteurs et des jurilinguistes. Le rédacteur est déjà suffisamment occupé à traduire les politiques législatives en forme législative, à gérer les aspects juridiques et rédactionnels de son dossier et à coordonner les rencontres, les consultations et les discussions associées à tout dossier législatif, sans devoir en plus se concentrer sur les aspects linguistiques du dossier, domaine dans lequel il n'est pas particulièrement qualifié de toute manière, même s'il a en général une bonne maîtrise de la langue dans laquelle il est appelé à rédiger des textes de loi.

Après 1978, il n'est pas exagéré de soutenir que les jurilinguistes sont devenus des collaborateurs indispensables dans le processus rédactionnel. Ils ont joué un rôle essentiel lors de la mise en place de la corédaction et la réalisation du Guide canadien de rédaction législative française. Sans leur apport majeur, il aurait été impossible de créer le style que l'on retrouve maintenant dans la version française des lois fédérales. Cet appui demeure toujours essentiel. C'est en étroite collaboration avec les jurilinguistes que les légistes peuvent assurer la qualité linguistique des textes de loi fédéraux. Les rédacteurs d'expérience ont bien sûr moins besoin de recourir aux services des jurilinguistes que les nouveaux venus. Le fait d'avoir travaillé pendant des années en étroite collaboration avec ces experts de la langue leur aura permis d'acquérir de bonnes connaissances linguistiques, mais pas au point de n'avoir plus besoin de recourir aux services des jurilinguistes, au moins sur une base ponctuelle.

Open Access This article is distributed under the terms of the Creative Commons Attribution License which permits any use, distribution, and reproduction in any medium, provided the original author(s) and the source are credited. 